CRYSTALLOGRAPHIC COMMUNICATIONS

ISSN 2056-9890

Received 28 June 2019

Accepted 8 July 2019

Edited by W. T. A. Harrison, University of Aberdeen, Scotland

Keywords: 1,2,4-triazolyl carboxylate; silver(I) metal-organic framework; hydrogen bonding; crystal structure.

CCDC reference: 1939097

Supporting information: this article has supporting information at journals.iucr.org/e

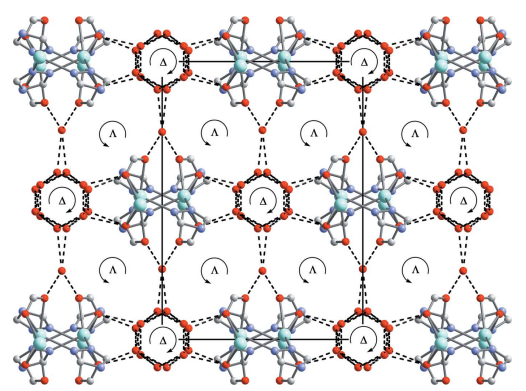

OPEN $\odot$ ACCESS

\section{Crystal structure of poly[[[ $\mu_{4}-3-(1,2,4-t r i a z o l-4-y l)-$ adamantane-1-carboxylato- $\left.\kappa^{5} N^{1}: N^{2}: O^{1}: O^{1}, O^{1}\right]$ - silver(I)] dihydrate]}

\author{
Ganna A. Senchyk, ${ }^{\mathrm{a} *}$ Harald Krautscheid ${ }^{\mathrm{b}}$ and Kostiantyn V. Domasevitch ${ }^{\mathrm{a}}$
}

anorganic Chemistry Department, Taras Shevchenko National University of Kyiv, Volodymyrska Street, 64, Kyiv 01033, Ukraine, and ${ }^{\mathbf{b}}$ Institut für Anorganische Chemie, Universitat Leipzig, Johannisallee 29, D-04103, Leipzig, Germany. *Correspondence e-mail: senchyk.ganna@gmail.com

The heterobifunctional organic ligand, 3-(1,2,4-triazol-4-yl)adamantane-1carboxylate (tr-ad-COO${ }^{-}$), was employed for the synthesis of the title silver(I) coordination polymer, $\left\{\left[\mathrm{Ag}\left(\mathrm{C}_{13} \mathrm{H}_{16} \mathrm{~N}_{3} \mathrm{O}_{2}\right)\right] \cdot 2 \mathrm{H}_{2} \mathrm{O}\right\}_{n}$, crystallizing in the rare orthorhombic $C 222_{1}$ space group. Alternation of the double $\mu_{2}-1,2,4$-triazole and $\mu_{2}-\eta^{2}: \eta^{1}-\mathrm{COO}^{-}$(chelating, bridging mode) bridges between $\mathrm{Ag}^{\mathrm{I}}$ cations supports the formation of sinusoidal coordination chains. The $\mathrm{Ag}^{\mathrm{I}}$ centers possess a distorted $\left\{\mathrm{N}_{2} \mathrm{O}_{3}\right\}$ square-pyramidal arrangement with $\tau_{5}=0.30$. The angular organic linkers connect the chains into a tetragonal framework with small channels along the $c$-axis direction occupied by water molecules of crystallization, which are interlinked via $\mathrm{O}-\mathrm{H} \cdots \mathrm{O}$ hydrogen bonds with carboxylate groups, leading to right- and left-handed helical dispositions.

\section{Chemical context}

Organic ligands, which contain two different functional groups, such as azole and carboxylic groups, attract attention in the context of the construction of unusual metal-organic frameworks (MOFs) including heterometallic architectures (Guillerm et al. 2014). Each ligand function is intended to introduce its coordination ability towards a metal center forming secondary building units (SBUs) based on its peculiarities. For instance, 1,2,4-triazoles (tr) typically serve as short $N, N$-bridges between two metal ions resulting in polynuclear units and chains (Wang et al. 2007, Murdock \& Jenkins 2014). In contrast, carboxylate groups offer a much broader variety of coordination modes: mono-, chelate-, bridging- and their combinations; and the number of connected metal ions may differ from one to four (Sun et al. 2004; Lu et al. 2014). As shown by Lincke et al. (2011, 2012), 1,2,4-triazolecarboxylate ligands are good candidates for the construction of microporous MOFs suitable for gas sorption and separation. Considering the heterofunctional $t r / C O O$ ligands, there are two possible roles for them to play. First, the 'separate' role, where $t r$ is responsible for di-, tri- or tetranuclear cluster formation, whereas the $\mathrm{COO}^{-}$group only occupies terminal (non-bridging) positions (Handke et al. 2014) or it can be involved in the separate coordination to metal centers. In this context, Chen et al. (2011) used 1,2,4-triazolyl isophthalate as a ligand in the synthesis of a series of $\mathrm{Ag}^{\mathrm{I}}-\mathrm{Ln}^{\mathrm{III}}$ heterometallic coordination polymers. Second, in the 'cooperative' role, $t r$ / $\mathrm{COO}$ serves as a heteroleptic bridge between the metal centers (Vasylevs'kyy et al. 2015). 


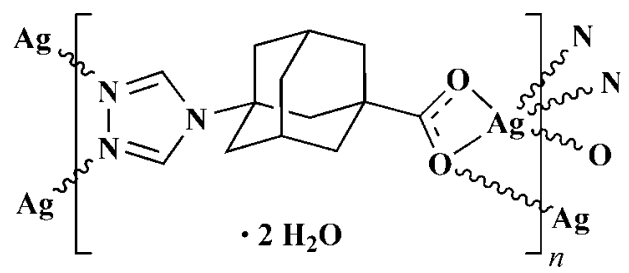

In present paper, we report the crystal structure of a new silver(I) coordination polymer $[\mathrm{Ag}(\text { tr-ad-COO })]_{n} \cdot 2 \mathrm{H}_{2} \mathrm{O}$ (I) based on the 1-(1,2,4-triazol-4-yl)-3-carboxyadamantane $\left(\mathrm{C}_{13} \mathrm{H}_{16} \mathrm{~N}_{3} \mathrm{O}_{2} ;\right.$ tr-ad-COOH) ligand.

\section{Structural commentary}

The title compound I crystallizes in the orthorhombic system with the uncommon space group $C 222_{1}$. The asymmetric unit contains one $\mathrm{Ag}^{\mathrm{I}}$ cation, one organic ligand and three distinct water molecules of crystallization, one of which (O5) is disordered over two adjacent sites (Fig. 1). The O3 water molecule is situated on a crystallographic twofold axis passing through the $\mathrm{O}$ atom, while the $\mathrm{O} 4$ water molecule is statistically disordered over two positions, both possessing an occupancy factor of 0.5. Thus, in the asymmetric unit, the total atom content sums up to two water molecules. The 1,2,4triazole functional group is coordinated by two $\mathrm{Ag}^{\mathrm{I}}$ centers as a $\mu_{2}-N, N$ bridge and the carboxylate group connects two $\mathrm{Ag}^{\mathrm{I}}$ centers in a chelating, bridging mode $\left(\mu_{2}-\eta^{2}: \eta^{1}\right)$, supporting the formation of sinusoidal chains with a periodicity of $13 \AA$.

In the case of compound $\mathbf{I}$ an unusual situation with alternation of double triazoles and double carboxylate bridges within the chain is observed. Thus, the $t r-a d-C O O^{-}$ligands act in a deprotonated form adopting a $\mu_{4}$-coordination modes (Fig. 2) that yields a three-dimensional tetragonal pattern with open channels along the $c$-axis direction (Fig. 3).

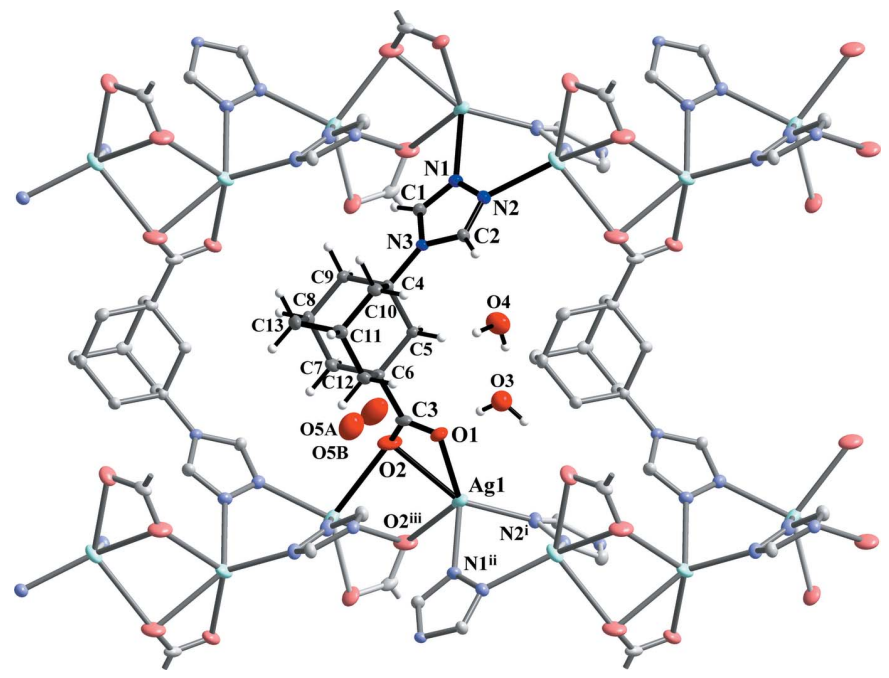

Figure 1

Fragment of the crystal structure of $\mathbf{I}$. The independent part of the structure is indicated with black bonds and displacement ellipsoids are drawn at the $50 \%$ probability level. [Symmetry codes: (i) $\frac{1}{2}-x,-\frac{1}{2}+y$, $\frac{1}{2}-z$, (ii) $\frac{1}{2}+x,-\frac{1}{2}+y, z$, (iii) $\left.x,-y, 1-z\right]$

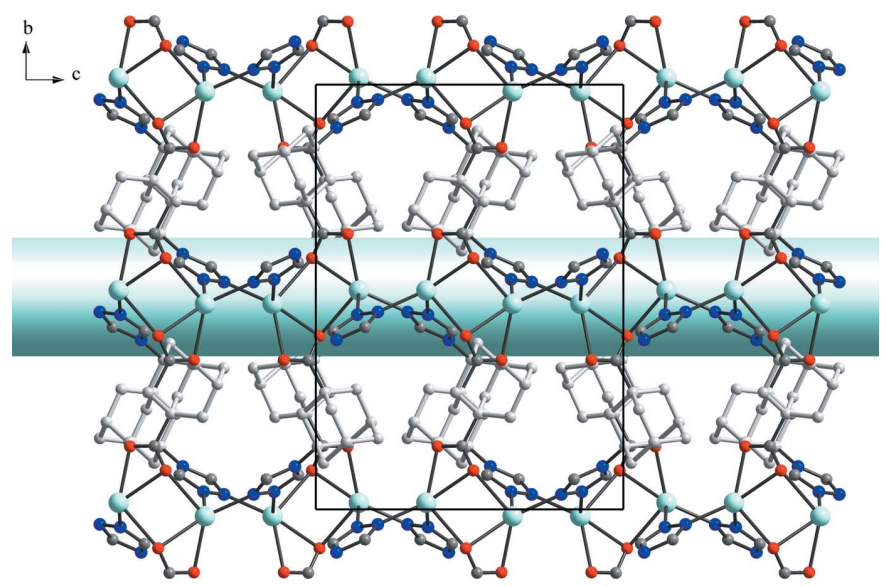

Figure 2

Projection on the $b c$ plane showing the interconnection of sinusoidal $\mathrm{Ag}^{\mathrm{I}}$ coordination chains by means of $t r-a d-\mathrm{COO}^{-}$organic ligands into a threedimensional framework.

The coordination environment of the $\mathrm{Ag}^{\mathrm{I}}$ cation is a very distorted $\left\{\mathrm{N}_{2} \mathrm{O}_{3}\right\}$ polyhedron with two $\mathrm{Ag}-\mathrm{N}$ (triazole)

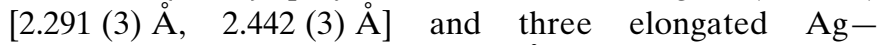
O(carboxylate) [2.437 (3)-2.703 (4) A] bonds (Table 1). The geometry of the five-coordinate center can be described by the geometric parameter $\tau_{5}$, which represents the degree of trigonality between two ideal structures - trigonal bipyramid

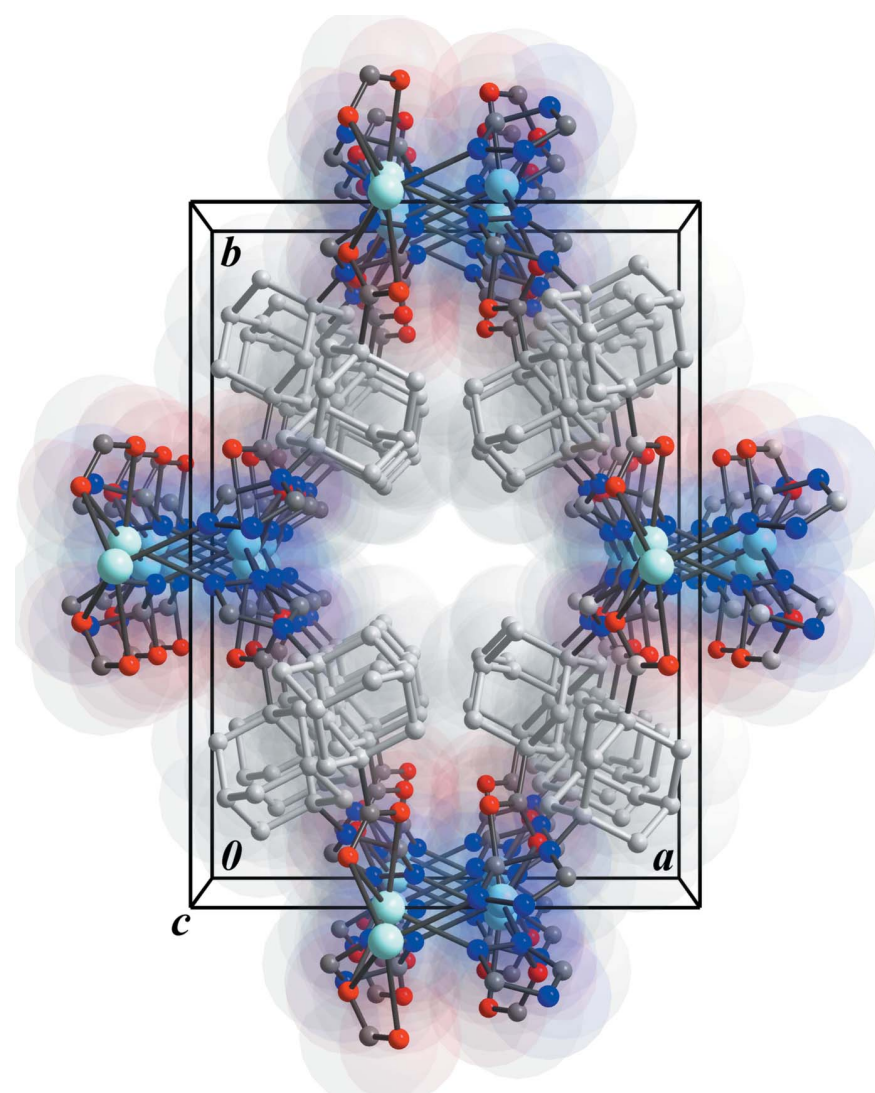

Figure 3

View of the channels along the $c$ axis in the structure of $\mathbf{I}$. 
Table 1

Selected geometric parameters $\left(\AA,^{\circ}\right)$.

\begin{tabular}{lrlr}
\hline $\mathrm{Ag} 1-\mathrm{N} 2^{\mathrm{i}}$ & $2.291(3)$ & $\mathrm{Ag} 1-\mathrm{O} 2$ & $2.571(4)$ \\
$\mathrm{Ag} 1-\mathrm{O} 1$ & $2.437(3)$ & $\mathrm{Ag} 1-\mathrm{O} 2^{\mathrm{iii}}$ & $2.703(4)$ \\
$\mathrm{Ag} 1-\mathrm{N} 1^{1 i}$ & $2.442(3)$ & & \\
& & & \\
$\mathrm{N} 2^{\mathrm{i}}-\mathrm{Ag} 1-\mathrm{O} 1$ & $125.93(11)$ & $\mathrm{N} 1^{\mathrm{ii}}-\mathrm{Ag} 1-\mathrm{O} 2$ & $121.93(12)$ \\
$\mathrm{N} 2^{\mathrm{i}}-\mathrm{Ag} 1-\mathrm{N} 1^{\mathrm{ii}}$ & $92.12(11)$ & $\mathrm{N} 2^{\mathrm{i}}-\mathrm{Ag} 1-\mathrm{O} 2^{\mathrm{iii}}$ & $101.35(11)$ \\
$\mathrm{O} 1-\mathrm{Ag} 1-\mathrm{N} 1^{\mathrm{ii}}$ & $106.84(11)$ & $\mathrm{O} 1-\mathrm{Ag} 1-\mathrm{O} 2^{\mathrm{iii}}$ & $128.04(11)$ \\
$\mathrm{N} 2^{\mathrm{i}}-\mathrm{Ag} 1-\mathrm{O} 2$ & $145.74(12)$ & $\mathrm{N} 1^{\mathrm{ii}}-\mathrm{Ag} 1-\mathrm{O} 2^{\mathrm{iii}}$ & $89.79(11)$ \\
$\mathrm{O} 1-\mathrm{Ag} 1-\mathrm{O} 2$ & $51.95(11)$ & $\mathrm{O} 2-\mathrm{Ag} 1-\mathrm{O} 2^{\mathrm{iii}}$ & $77.22(13)$ \\
\hline
\end{tabular}

Symmetry codes: (i) $-x+\frac{1}{2}, y-\frac{1}{2},-z+\frac{1}{2}$; (ii) $x+\frac{1}{2}, y-\frac{1}{2}, z$; (iii) $x,-y,-z+1$.

$\left(\tau_{5}=1\right)$ and square pyramid $\left(\tau_{5}=0\right)$ (Addison et al., 1984). In compound $\mathbf{I}$, the Ag1 center has $\tau_{5}=0.30$, indicating a significantly distorted square-pyramidal geometry.

\section{Supramolecular features}

The water guest molecules inside the [001] channels are responsible for the extended hydrogen-bonding network (Table 2). Together with the $-\mathrm{COO}^{-}$groups, they are organized into two types of helices along the $c$ axis - smaller righthanded (A in Fig. 4) and bigger left-handed (B in Fig. 4). In addition, weak $\mathrm{C}-\mathrm{H}_{\text {(triazole })} \cdot \mathrm{O} 1_{(\mathrm{COO})}$ and $\mathrm{C}-\mathrm{H}_{\text {(triazo- }}$ le)... 04 (water) contacts are observed. The packig is shown in Fig. 5.

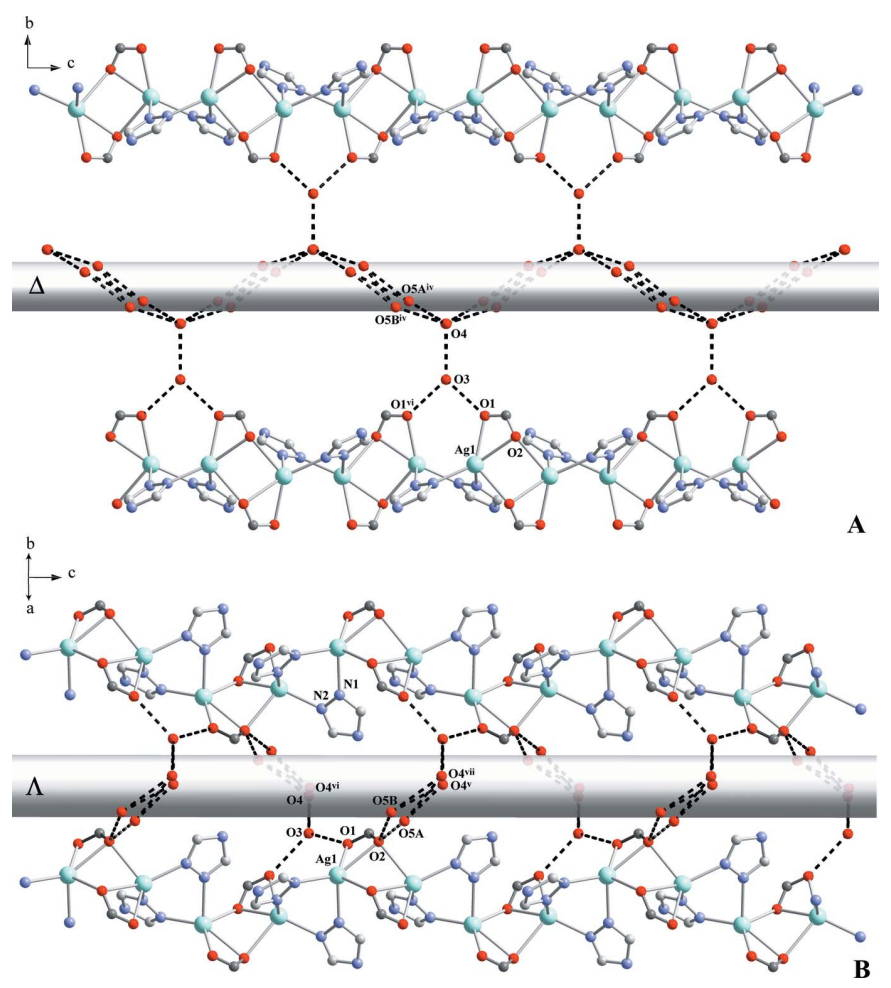

Figure 4

Hydrogen-bonding scheme between water molecules and $\mathrm{COO}^{-}$groups showing the formation of right-handed (A) and left-handed (B) helices. Adamantyl fragments are omitted for clarity. [Symmetry codes: (iv) $\frac{1}{2}+x$, $\frac{1}{2}-y, 1-z ;(\mathrm{v})-\frac{1}{2}+x, \frac{1}{2}-y, 1-z ;(\mathrm{vi}) 1-x, y, \frac{1}{2}-z ;(\mathrm{vii}) \frac{1}{2}-x, \frac{1}{2}-y$, $\frac{1}{2}+z$.]
Table 2

Hydrogen-bond geometry $\left(\AA,^{\circ}\right)$.

\begin{tabular}{|c|c|c|c|c|}
\hline$D-\mathrm{H} \cdots A$ & $D-\mathrm{H}$ & $\mathrm{H} \cdots A$ & $D \cdots A$ & $D-\mathrm{H} \cdots A$ \\
\hline $\mathrm{O} 3-\mathrm{H} 1 W \cdots \mathrm{O} 1$ & 0.85 & 1.97 & $2.818(5)$ & 171 \\
\hline $\mathrm{O} 4-\mathrm{H} 2 W \cdots \mathrm{O} 3$ & 0.85 & 1.92 & $2.746(10)$ & 163 \\
\hline $\mathrm{O} 4-\mathrm{H} 3 W \cdots \mathrm{O} 5 A^{\mathrm{iv}}$ & 0.85 & 1.81 & $2.56(2)$ & 147 \\
\hline $\mathrm{O} 4-\mathrm{H} 3 W \cdots \mathrm{O} 5 B^{\mathrm{iv}}$ & 0.85 & 2.11 & $2.83(3)$ & 143 \\
\hline $\mathrm{C} 1-\mathrm{H} 1 \cdots \mathrm{O} 1^{\mathrm{v}}$ & 0.94 & 2.43 & $3.336(5)$ & 162 \\
\hline $\mathrm{C} 2-\mathrm{H} 2 \cdots \mathrm{O} 4^{\mathrm{vi}}$ & 0.94 & 2.58 & $3.516(12)$ & 171 \\
\hline
\end{tabular}

Symmetry codes: (iv) $x+\frac{1}{2},-y+\frac{1}{2},-z+1 ; \quad$ (v) $x-\frac{1}{2},-y+\frac{1}{2},-z+1$; $-x+1, y,-z+\frac{1}{2}$.

\section{Synthesis and crystallization}

1-(1,2,4-Triazol-4-yl)-3-carboxyadamantane (tr-ad-COOH) was synthesized by refluxing 3-amino-adamantane-1-carboxylic acid (Wanka et al., 2007) (3.00 g, $15.4 \mathrm{mmol}$ ) and dimethylformamide azine $(5.46 \mathrm{~g}, 38.5 \mathrm{mmol})$ in the presence of toluenesulfonic acid monohydrate $(0.44 \mathrm{~g}, 2.3 \mathrm{mmol})$ as catalyst in DMF $(30 \mathrm{ml})$. Yield $=63 \%$.

The synthesis of I was carried out under hydrothermal conditions as follows. A mixture of $\mathrm{AgNO}_{3}(17.0 \mathrm{mg}$, $0.100 \mathrm{mmol}$ ), tr-ad-COOH (12.4 mg, $0.050 \mathrm{mmol})$ and $5 \mathrm{ml}$ of water was added into a Teflon vessel, which was sealed and heated at $413 \mathrm{~K}$ for $24 \mathrm{~h}$ and slowly cooled to room temperature over $48 \mathrm{~h}$, yielding colourless needles of I (yield $13.3 \mathrm{mg}, 68 \%$ ).

\section{Refinement}

Crystal data, data collection and structure refinement details are summarized in Table 3. O4 lies adjacent to a crystallographic twofold axis and is statistically disordered over two positions ( $\mathrm{O} 4 \cdots \mathrm{O} 4=0.60 \AA$ ) and $\mathrm{O} 5$ is statistically disordered over adjacent locations (O5A $\cdots \mathrm{O} 5 B=0.77 \AA)$. $\mathrm{CH}$ hydrogen atoms were positioned geometrically and refined as riding,

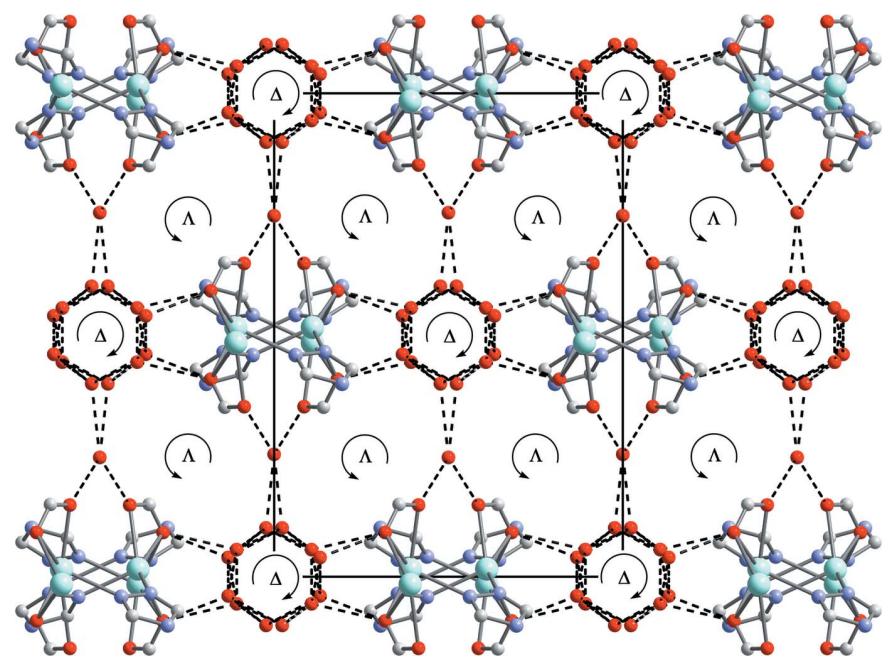

Figure 5

The packing of the right- and left-handed helices in the crystal structure of I (top view). Adamantyl fragments are omitted for clarity. 
with $\mathrm{C}-\mathrm{H}=0.94 \AA$ (triazole); $\mathrm{C}-\mathrm{H}=0.98 \AA$ (adamantane $\left.\mathrm{CH}_{2}\right) ; \mathrm{C}-\mathrm{H}=0.99 \AA$ (adamantane $\mathrm{CH}$ ) and with $U_{\text {iso }}(\mathrm{H})=$ $1.2 U_{\text {eq }}(\mathrm{C}) . \mathrm{OH}$ hydrogen atoms were located and then refined with $\mathrm{O}-\mathrm{H}=0.85 \AA\left(\mathrm{H}_{2} \mathrm{O}\right)$ and with $U_{\text {iso }}(\mathrm{H})=1.5 U_{\text {eq }}(\mathrm{O})$. For one of the disordered water molecules, the $\mathrm{H}$ atoms were not located.

\section{Funding information}

Funding for this research was provided by: Ministry of Education and Science of Ukraine (grant No. 19BF037-05).

\section{References}

Addison, A. W., Rao, T. N., Reedijk, J., van Rijn, J. \& Verschoor, G. C. (1984). J. Chem. Soc. Dalton Trans. pp. 1349-1356.

Brandenburg, K. (1999). DIAMOND. Crystal Impact GbR, Bonn, Germany.

Chen, M., Chen, M.-S., Okamura, T., Lv, M.-F., Sun, W.-Y. \& Ueyama, N. (2011). CrystEngComm, 13, 3801-3810.

Farrugia, L. J. (2012). J. Appl. Cryst. 45, 849-854.

Guillerm, V., Kim, D., Eubank, J. F., Luebke, R., Liu, X., Adil, K., Lah, M. S. \& Eddaoudi, M. (2014). Chem. Soc. Rev. 43, 6141-6172.

Handke, M., Weber, H., Lange, M., Möllmer, J., Lincke, J., Gläser, R., Staudt, R. \& Krautscheid, H. (2014). Inorg. Chem. 53, 7599-7607.

Lincke, J., Lässig, D., Kobalz, M., Bergmann, J., Handke, M., Möllmer, J., Lange, M., Roth, C., Möller, A., Staudt, R. \& Krautscheid, H. (2012). Inorg. Chem. 51, 7579-7586.

Lincke, J., Lässig, D., Moellmer, J., Reichenbach, C., Puls, A., Moeller, A., Gläser, R., Kalies, G., Staudt, R. \& Krautscheid, H. (2011). Microporous Mesoporous Mater. 142, 62-69.

Lu, W., Wei, Z., Gu, Z.-Y., Liu, T.-F., Park, J., Park, J., Tian, J., Zhang, M., Zhang, Q., Gentle, T. III, Bosch, M. \& Zhou, H.-C. (2014). Chem. Soc. Rev. 43, 5561-5593.

Murdock, C. R. \& Jenkins, D. M. (2014). J. Am. Chem. Soc. 136, 10983-10988.

Parsons, S., Flack, H. D. \& Wagner, T. (2013). Acta Cryst. B69, 249259.

Sheldrick, G. M. (2008). Acta Cryst. A64, 112-122.

Sheldrick, G. M. (2015). Acta Cryst. C71, 3-8.

Stoe \& Cie (1999). X-SHAPE. Stoe \& Cie GmbH, Darmstadt, Germany.

Stoe \& Cie (2000). IPDS Software. Stoe \& Cie GmbH, Darmstadt, Germany.

Stoe \& Cie (2001). X-RED. Stoe \& Cie GmbH, Darmstadt, Germany.
Table 3

Experimental details.

\begin{tabular}{|c|c|}
\hline \multicolumn{2}{|l|}{ Crystal data } \\
\hline Chemical formula & {$\left[\mathrm{Ag}\left(\mathrm{C}_{13} \mathrm{H}_{16} \mathrm{~N}_{3} \mathrm{O}_{2}\right)\right] \cdot 2 \mathrm{H}_{2} \mathrm{O}$} \\
\hline$M_{\mathrm{r}}$ & 390.19 \\
\hline Crystal system, space group & Orthorhombic, $C 222_{1}$ \\
\hline Temperature $(\mathrm{K})$ & 213 \\
\hline$a, b, c(\AA)$ & $\begin{array}{l}12.9321(9), 17.9056(10), \\
12.9695(9)\end{array}$ \\
\hline$V\left(\AA^{3}\right)$ & $3003.2(3)$ \\
\hline$Z$ & 8 \\
\hline Radiation type & Mo $K \alpha$ \\
\hline$\mu\left(\mathrm{mm}^{-1}\right)$ & 1.36 \\
\hline Crystal size $(\mathrm{mm})$ & $0.22 \times 0.18 \times 0.16$ \\
\hline \multicolumn{2}{|l|}{ Data collection } \\
\hline Diffractometer & Stoe Image plate diffraction system \\
\hline Absorption correction & $\begin{array}{l}\text { Numerical }[X-R E D \text { (Stoe \& Cie, } \\
\text { 2001) and } X \text {-SHAPE (Stoe \& } \\
\text { Cie, 1999)] }\end{array}$ \\
\hline$T_{\min }, T_{\max }$ & $0.649,0.689$ \\
\hline $\begin{array}{l}\text { No. of measured, independent and } \\
\text { observed }[I>2 \sigma(I)] \text { reflections }\end{array}$ & $13596,3617,3135$ \\
\hline$R_{\text {int }}$ & 0.028 \\
\hline$(\sin \theta / \lambda)_{\max }\left(\AA^{-1}\right)$ & 0.661 \\
\hline \multicolumn{2}{|l|}{ Refinement } \\
\hline$R\left[F^{2}>2 \sigma\left(F^{2}\right)\right], w R\left(F^{2}\right), S$ & $0.025,0.058,0.97$ \\
\hline No. of reflections & 3617 \\
\hline No. of parameters & 204 \\
\hline $\mathrm{H}$-atom treatment & $\mathrm{H}$-atom parameters constrained \\
\hline$\Delta \rho_{\max }, \Delta \rho_{\min }\left(\mathrm{e} \AA^{-3}\right)$ & $0.89,-0.94$ \\
\hline Absolute structure & $\begin{array}{l}\text { Flack } x \text { determined using } 1289 \\
\text { quotients }\left[\left(I^{+}\right)-\left(I^{-}\right)\right] /\left[\left(I^{+}\right)+\left(I^{-}\right)\right] \\
\text {(Parsons et al., 2013) }\end{array}$ \\
\hline Absolute structure parameter & $-0.060(9)$ \\
\hline
\end{tabular}

Computer programs: IPDS Software (Stoe \& Cie, 2000), SHELXS97 (Sheldrick, 2008), SHELXL2014 (Sheldrick, 2015), DIAMOND (Brandenburg, 1999) and WinGX (Farrugia, 2012).

Sun, D., Cao, R., Bi, W., Weng, J., Hong, M. \& Liang, Y. (2004). Inorg. Chim. Acta, 357, 991-1001.

Vasylevs'kyy, S. I., Lysenko, A. B., Krautscheid, H., Karbowiak, M., Rusanov, E. B. \& Domasevitch, K. V. (2015). Inorg. Chem. Commun. 62, 51-54.

Wang, Y., Ding, B., Cheng, P., Liao, D.-Z. \& Yan, S.-P. (2007). Inorg. Chem. 46, 2002-2010.

Wanka, L., Cabrele, C., Vanejews, M. \& Schreiner, P. R. (2007) Eur. J. Org. Chem. pp. 1474-1490. 


\section{supporting information}

Acta Cryst. (2019). E75, 1145-1148 [https://doi.org/10.1107/S2056989019009708]

Crystal structure of poly[[[ $\mu_{4}-3-(1,2,4$-triazol-4-yl)adamantane-1-carboxylato$\left.\kappa^{5} N^{1}: N^{2}: O^{1}: O^{1}, O^{1}\right]$ silver(I)] dihydrate]

\section{Ganna A. Senchyk, Harald Krautscheid and Kostiantyn V. Domasevitch}

Computing details

Data collection: IPDS Software (Stoe \& Cie, 2000); cell refinement: IPDS Software (Stoe \& Cie, 2000); data reduction: IPDS Software (Stoe \& Cie, 2000); program(s) used to solve structure: SHELXS97 (Sheldrick, 2008); program(s) used to refine structure: SHELXL2014 (Sheldrick, 2015); molecular graphics: DIAMOND (Brandenburg, 1999); software used to prepare material for publication: $\operatorname{Win} G X$ (Farrugia, 2012).

Poly[[[ $\mu_{4}-3-\left(1,2,4-\right.$ triazol-4-yl)adamantane-1-carboxylato- $\left.\kappa^{5} N^{1}: N^{2}: O^{1}: O^{1}, O^{1}\right]$ silver(I)] dihydrate]

\section{Crystal data}

$\left[\mathrm{Ag}\left(\mathrm{C}_{13} \mathrm{H}_{16} \mathrm{~N}_{3} \mathrm{O}_{2}\right)\right] \cdot 2 \mathrm{H}_{2} \mathrm{O}$

$M_{r}=390.19$

Orthorhombic, $C 222_{1}$

$a=12.9321$ (9) $\AA$

$b=17.9056(10) \AA$

$c=12.9695(9) \AA$

$V=3003.2(3) \AA^{3}$

$Z=8$

$F(000)=1584$

\section{Data collection}

Stoe Image plate diffraction system diffractometer

$\varphi$ oscillation scans

Absorption correction: numerical

[X-RED (Stoe \& Cie, 2001) and X-SHAPE (Stoe \& Cie, 1999)]

$T_{\text {min }}=0.649, T_{\max }=0.689$

13596 measured reflections

\section{Refinement}

Refinement on $F^{2}$

Least-squares matrix: full

$R\left[F^{2}>2 \sigma\left(F^{2}\right)\right]=0.025$

$w R\left(F^{2}\right)=0.058$

$S=0.97$

3617 reflections

204 parameters

0 restraints

Hydrogen site location: mixed

$\mathrm{H}$-atom parameters constrained
$D_{\mathrm{x}}=1.726 \mathrm{Mg} \mathrm{m}^{-3}$

Mo $K \alpha$ radiation, $\lambda=0.71073 \AA$

Cell parameters from 13596 reflections

$\theta=2.8-28.0^{\circ}$

$\mu=1.36 \mathrm{~mm}^{-1}$

$T=213 \mathrm{~K}$

Needle, colourless

$0.22 \times 0.18 \times 0.16 \mathrm{~mm}$

3617 independent reflections

3135 reflections with $I>2 \sigma(I)$

$R_{\text {int }}=0.028$

$\theta_{\text {max }}=28.0^{\circ}, \theta_{\min }=2.8^{\circ}$

$h=-17 \rightarrow 17$

$k=-22 \rightarrow 23$

$l=-17 \rightarrow 17$

$$
\begin{aligned}
& w=1 /\left[\sigma^{2}\left(F_{\mathrm{o}}{ }^{2}\right)+(0.037 P)^{2}\right] \\
& \text { where } P=\left(F_{\mathrm{o}}{ }^{2}+2 F_{\mathrm{c}}{ }^{2}\right) / 3 \\
& (\Delta / \sigma)_{\max }=0.001 \\
& \Delta \rho_{\max }=0.89 \mathrm{e} \AA^{-3} \\
& \Delta \rho_{\min }=-0.94 \mathrm{e} \AA^{-3}
\end{aligned}
$$

Absolute structure: Flack $x$ determined using 1289 quotients $\left[\left(I^{+}\right)-\left(I^{-}\right)\right] /\left[\left(I^{+}\right)+\left(I^{-}\right)\right]$(Parsons et al., 2013)

Absolute structure parameter: -0.060 (9) 


\section{Special details}

Geometry. All esds (except the esd in the dihedral angle between two 1.s. planes) are estimated using the full covariance matrix. The cell esds are taken into account individually in the estimation of esds in distances, angles and torsion angles; correlations between esds in cell parameters are only used when they are defined by crystal symmetry. An approximate (isotropic) treatment of cell esds is used for estimating esds involving l.s. planes.

Fractional atomic coordinates and isotropic or equivalent isotropic displacement parameters $\left(\AA^{2}\right)$

\begin{tabular}{|c|c|c|c|c|c|}
\hline & $x$ & $y$ & $z$ & $U_{\text {iso }} * / U_{\text {eq }}$ & Occ. $(<1)$ \\
\hline $\mathrm{Ag} 1$ & $0.39032(3)$ & $0.01581(2)$ & $0.35981(2)$ & $0.04198(10)$ & \\
\hline $\mathrm{O} 1$ & $0.4141(3)$ & $0.14822(17)$ & $0.3962(2)$ & $0.0474(8)$ & \\
\hline $\mathrm{O} 2$ & $0.3185(3)$ & $0.09152(19)$ & $0.5123(3)$ & $0.0581(9)$ & \\
\hline N1 & $0.0616(2)$ & $0.45845(17)$ & $0.3648(3)$ & $0.0318(6)$ & \\
\hline $\mathrm{N} 2$ & $0.1451(2)$ & $0.46474(17)$ & $0.2982(2)$ & $0.0316(7)$ & \\
\hline N3 & $0.1946(2)$ & $0.39903(16)$ & $0.4325(2)$ & $0.0231(6)$ & \\
\hline $\mathrm{C} 1$ & $0.0930(3)$ & $0.4190(2)$ & $0.4435(3)$ & $0.0282(8)$ & \\
\hline $\mathrm{H} 1$ & 0.0514 & 0.4060 & 0.5001 & $0.034^{*}$ & \\
\hline $\mathrm{C} 2$ & $0.2230(3)$ & $0.4290(2)$ & $0.3398(2)$ & $0.0276(7)$ & \\
\hline $\mathrm{H} 2$ & 0.2890 & 0.4245 & 0.3102 & $0.033^{*}$ & \\
\hline $\mathrm{C} 3$ & $0.3583(3)$ & $0.1495(2)$ & 0.4758 & $0.0339(9)$ & \\
\hline $\mathrm{C} 4$ & $0.2574(2)$ & $0.3521(2)$ & $0.5055(3)$ & $0.0219(7)$ & \\
\hline $\mathrm{C} 5$ & $0.2762(3)$ & $0.27539(19)$ & $0.4550(3)$ & $0.0234(7)$ & \\
\hline $\mathrm{H} 5 \mathrm{~A}$ & 0.3139 & 0.2819 & 0.3900 & $0.028^{*}$ & \\
\hline $\mathrm{H} 5 \mathrm{~B}$ & 0.2098 & 0.2515 & 0.4396 & $0.028^{*}$ & \\
\hline C6 & 0.3398 & $0.2253(2)$ & 0.5293 & $0.0260(7)$ & \\
\hline $\mathrm{C} 7$ & $0.2807(3)$ & $0.2174(2)$ & 0.6319 (3) & $0.0333(8)$ & \\
\hline $\mathrm{H} 7 \mathrm{~A}$ & 0.2140 & 0.1929 & 0.6196 & $0.040^{*}$ & \\
\hline $\mathrm{H} 7 \mathrm{~B}$ & 0.3206 & 0.1862 & 0.6796 & $0.040^{*}$ & \\
\hline $\mathrm{C} 8$ & $0.2626(3)$ & $0.2948(2)$ & $0.6802(3)$ & $0.0333(9)$ & \\
\hline H8 & 0.2247 & 0.2887 & 0.7460 & $0.040^{*}$ & \\
\hline $\mathrm{C} 9$ & $0.1977(3)$ & $0.3427(2)$ & $0.6064(3)$ & $0.0278(8)$ & \\
\hline H9A & 0.1311 & 0.3184 & 0.5931 & $0.033^{*}$ & \\
\hline H9B & 0.1843 & 0.3917 & 0.6374 & $0.033^{*}$ & \\
\hline $\mathrm{C} 10$ & $0.3616(3)$ & $0.3907(2)$ & 0.5253 & $0.0287(8)$ & \\
\hline H10A & 0.3502 & 0.4401 & 0.5556 & $0.034 *$ & \\
\hline H10B & 0.3990 & 0.3970 & 0.4601 & $0.034^{*}$ & \\
\hline C11 & 0.4256 & $0.3416(2)$ & $0.5999(3)$ & $0.0326(8)$ & \\
\hline H11 & 0.4930 & 0.3659 & 0.6135 & $0.039^{*}$ & \\
\hline $\mathrm{C} 12$ & 0.4439 & $0.2646(2)$ & $0.5505(3)$ & $0.0294(8)$ & \\
\hline H12A & 0.4859 & 0.2338 & 0.5968 & $0.035^{*}$ & \\
\hline H12B & 0.4819 & 0.2707 & 0.4857 & $0.035^{*}$ & \\
\hline C13 & $0.3663(3)$ & $0.3327(2)$ & $0.7016(3)$ & $0.0384(10)$ & \\
\hline H13A & 0.4069 & 0.3025 & 0.7499 & $0.046^{*}$ & \\
\hline H13B & 0.3547 & 0.3818 & 0.7328 & $0.046^{*}$ & \\
\hline $\mathrm{O} 3$ & 0.5000 & $0.2468(3)$ & 0.2500 & $0.0630(13)$ & \\
\hline $\mathrm{H} 1 \mathrm{~W}$ & 0.4787 & 0.2190 & 0.2988 & $0.094^{*}$ & \\
\hline $\mathrm{O} 4$ & $0.5231(6)$ & $0.3992(4)$ & $0.2479(18)$ & 0.068 & 0.5 \\
\hline $\mathrm{H} 2 \mathrm{~W}$ & 0.5153 & 0.3530 & 0.2348 & $0.102 *$ & 0.5 \\
\hline
\end{tabular}




\begin{tabular}{llllll} 
H3W & 0.5724 & 0.4038 & 0.2909 & $0.102^{*}$ & 0.5 \\
O5A & $0.1318(13)$ & $0.0398(10)$ & $0.6097(11)$ & $0.096(4)$ & 0.5 \\
O5B & $0.1073(12)$ & $0.0560(11)$ & $0.5604(12)$ & $0.100(5)$ & 0.5 \\
\hline
\end{tabular}

Atomic displacement parameters $\left(\AA^{2}\right)$

\begin{tabular}{lllllll}
\hline & $U^{11}$ & $U^{22}$ & $U^{33}$ & $U^{12}$ & $U^{13}$ & $U^{23}$ \\
\hline Ag1 & $0.04963(17)$ & $0.03609(16)$ & $0.04023(15)$ & $0.01122(15)$ & $-0.00959(15)$ & $-0.01749(13)$ \\
O1 & $0.063(2)$ & $0.0348(16)$ & $0.0446(17)$ & $0.0145(14)$ & $-0.0012(14)$ & $-0.0141(12)$ \\
O2 & $0.064(2)$ & $0.0231(18)$ & $0.087(3)$ & $0.0013(17)$ & $0.005(2)$ & $-0.0047(15)$ \\
N1 & $0.0325(15)$ & $0.0318(15)$ & $0.0311(14)$ & $0.0098(12)$ & $-0.0019(15)$ & $-0.0012(14)$ \\
N2 & $0.0379(17)$ & $0.0292(17)$ & $0.0275(14)$ & $0.0059(13)$ & $-0.0028(12)$ & $0.0018(12)$ \\
N3 & $0.0255(15)$ & $0.0201(15)$ & $0.0239(14)$ & $0.0042(12)$ & $-0.0023(11)$ & $0.0004(11)$ \\
C1 & $0.0258(19)$ & $0.0321(19)$ & $0.0266(17)$ & $0.0057(15)$ & $0.0039(14)$ & $0.0016(13)$ \\
C2 & $0.0290(17)$ & $0.0307(19)$ & $0.0231(17)$ & $0.0050(15)$ & $0.0002(13)$ & $0.0037(13)$ \\
C3 & $0.037(2)$ & $0.019(2)$ & $0.045(2)$ & $0.0076(16)$ & $-0.0134(16)$ & $-0.0061(17)$ \\
C4 & $0.0260(15)$ & $0.022(2)$ & $0.0176(13)$ & $0.0035(15)$ & $-0.0030(14)$ & $0.0009(11)$ \\
C5 & $0.0261(16)$ & $0.0211(17)$ & $0.0229(15)$ & $0.0034(14)$ & $-0.0023(13)$ & $-0.0028(13)$ \\
C6 & $0.0317(18)$ & $0.0184(17)$ & $0.0280(17)$ & $0.0040(15)$ & $-0.0026(13)$ & $-0.0024(13)$ \\
C7 & $0.0394(18)$ & $0.0290(19)$ & $0.0315(18)$ & $0.0046(15)$ & $0.0011(17)$ & $0.0080(16)$ \\
C8 & $0.042(2)$ & $0.039(2)$ & $0.0196(15)$ & $0.0089(17)$ & $0.0015(15)$ & $0.0045(14)$ \\
C9 & $0.0321(19)$ & $0.0278(19)$ & $0.0235(16)$ & $0.0076(15)$ & $0.0039(13)$ & $0.0010(13)$ \\
C10 & $0.0305(19)$ & $0.0208(19)$ & $0.0349(19)$ & $-0.0023(14)$ & $-0.0063(13)$ & $-0.0024(15)$ \\
C11 & $0.0323(19)$ & $0.0275(19)$ & $0.0380(19)$ & $0.0011(15)$ & $-0.0147(15)$ & $-0.0050(15)$ \\
C12 & $0.0272(18)$ & $0.029(2)$ & $0.0321(19)$ & $0.0056(16)$ & $-0.0059(14)$ & $-0.0003(15)$ \\
C13 & $0.052(3)$ & $0.037(2)$ & $0.0266(17)$ & $0.0087(18)$ & $-0.0121(16)$ & $-0.0052(15)$ \\
O3 & $0.067(3)$ & $0.060(3)$ & $0.062(3)$ & 0.000 & $0.001(3)$ & 0.000 \\
O4 & $0.051(9)$ & $0.068(4)$ & $0.085(5)$ & $-0.008(4)$ & $-0.018(10)$ & $0.000(6)$ \\
O5A & $0.089(10)$ & $0.109(9)$ & $0.091(10)$ & $-0.005(7)$ & $0.009(7)$ & $-0.018(8)$ \\
O5B & $0.060(7)$ & $0.128(12)$ & $0.110(11)$ & $0.003(8)$ & $-0.005(8)$ & $-0.045(10)$ \\
& & & & & & \\
& & & & & &
\end{tabular}

Geometric parameters $\left(\AA,{ }^{o}\right)$

\begin{tabular}{llll}
\hline $\mathrm{Ag} 1-\mathrm{N} 2^{\mathrm{i}}$ & $2.291(3)$ & $\mathrm{C} 6-\mathrm{C} 12$ & $1.544(5)$ \\
$\mathrm{Ag} 1-\mathrm{O} 1$ & $2.437(3)$ & $\mathrm{C} 7-\mathrm{C} 8$ & $1.538(5)$ \\
$\mathrm{Ag} 1-\mathrm{N} 1^{\mathrm{ii}}$ & $2.442(3)$ & $\mathrm{C} 7-\mathrm{H} 7 \mathrm{~A}$ & 0.9800 \\
$\mathrm{Ag} 1-\mathrm{O} 2$ & $2.571(4)$ & $\mathrm{C} 7-\mathrm{H} 7 \mathrm{~B}$ & 0.9800 \\
$\mathrm{Ag} 1-\mathrm{O} 2^{\mathrm{iii}}$ & $2.703(4)$ & $\mathrm{C} 8-\mathrm{C} 13$ & $1.529(6)$ \\
$\mathrm{O} 1-\mathrm{C} 3$ & $1.259(5)$ & $\mathrm{C} 8-\mathrm{C} 9$ & $1.536(5)$ \\
$\mathrm{O} 2-\mathrm{C} 3$ & $1.251(5)$ & $\mathrm{C} 8-\mathrm{H} 8$ & 0.9900 \\
$\mathrm{~N} 1-\mathrm{C} 1$ & $1.305(5)$ & $\mathrm{C} 9-\mathrm{H} 9 \mathrm{~A}$ & 0.9800 \\
$\mathrm{~N} 1-\mathrm{N} 2$ & $1.387(4)$ & $\mathrm{C} 9-\mathrm{H} 9 \mathrm{~B}$ & 0.9800 \\
$\mathrm{~N} 1-\mathrm{Ag} 1^{\mathrm{iv}}$ & $2.442(3)$ & $\mathrm{C} 10-\mathrm{C} 11$ & $1.548(5)$ \\
$\mathrm{N} 2-\mathrm{C} 2$ & $1.310(5)$ & $\mathrm{C} 10-\mathrm{H} 10 \mathrm{~A}$ & 0.9800 \\
$\mathrm{~N} 2-\mathrm{Ag} 1^{\mathrm{v}}$ & $2.291(3)$ & $\mathrm{C} 10-\mathrm{H} 10 \mathrm{~B}$ & 0.9800 \\
$\mathrm{~N} 3-\mathrm{C} 2$ & $1.366(4)$ & $\mathrm{C} 11-\mathrm{C} 13$ & $1.533(6)$ \\
$\mathrm{N} 3-\mathrm{C} 1$ & $1.369(5)$ & $\mathrm{C} 11-\mathrm{C} 12$ & $1.538(5)$ \\
$\mathrm{N} 3-\mathrm{C} 4$ & $1.505(4)$ & $\mathrm{C} 11-\mathrm{H} 11$ & 0.9900
\end{tabular}




\begin{tabular}{|c|c|c|c|}
\hline $\mathrm{C} 1-\mathrm{H} 1$ & 0.9400 & $\mathrm{C} 12-\mathrm{H} 12 \mathrm{~A}$ & 0.9800 \\
\hline $\mathrm{C} 2-\mathrm{H} 2$ & 0.9400 & $\mathrm{C} 12-\mathrm{H} 12 \mathrm{~B}$ & 0.9800 \\
\hline $\mathrm{C} 3-\mathrm{C} 6$ & $1.544(5)$ & $\mathrm{C} 13-\mathrm{H} 13 \mathrm{~A}$ & 0.9800 \\
\hline $\mathrm{C} 4-\mathrm{C} 9$ & $1.530(5)$ & $\mathrm{C} 13-\mathrm{H} 13 \mathrm{~B}$ & 0.9800 \\
\hline $\mathrm{C} 4-\mathrm{C} 10$ & $1.535(5)$ & $\mathrm{O} 3-\mathrm{H} 1 \mathrm{~W}$ & 0.8500 \\
\hline $\mathrm{C} 4-\mathrm{C} 5$ & $1.541(5)$ & $\mathrm{O} 4-\mathrm{O} 4^{\mathrm{vi}}$ & $0.601(16)$ \\
\hline $\mathrm{C} 5-\mathrm{C} 6$ & $1.552(5)$ & $\mathrm{O} 4-\mathrm{H} 2 \mathrm{~W}$ & 0.8500 \\
\hline $\mathrm{C} 5-\mathrm{H} 5 \mathrm{~A}$ & 0.9800 & $\mathrm{O} 4-\mathrm{H} 3 \mathrm{~W}$ & 0.8500 \\
\hline C5-H5B & 0.9800 & $\mathrm{O} 5 \mathrm{~A}-\mathrm{O} 5 \mathrm{~B}$ & $0.770(16)$ \\
\hline $\mathrm{C} 6-\mathrm{C} 7$ & $1.542(5)$ & & \\
\hline $\mathrm{N} 2 \mathrm{i}-\mathrm{Ag} 1-\mathrm{O} 1$ & $125.93(11)$ & $\mathrm{C} 3-\mathrm{C} 6-\mathrm{C} 5$ & $108.1(3)$ \\
\hline $\mathrm{N} 2^{\mathrm{i}}-\mathrm{Ag} 1-\mathrm{N} 1^{\mathrm{ii}}$ & $92.12(11)$ & $\mathrm{C} 8-\mathrm{C} 7-\mathrm{C} 6$ & $110.1(3)$ \\
\hline $\mathrm{O} 1-\mathrm{Ag} 1-\mathrm{N} 1^{\mathrm{ii}}$ & $106.84(11)$ & $\mathrm{C} 8-\mathrm{C} 7-\mathrm{H} 7 \mathrm{~A}$ & 109.6 \\
\hline $\mathrm{N} 2 \mathrm{i}-\mathrm{Ag} 1-\mathrm{O} 2$ & $145.74(12)$ & $\mathrm{C} 6-\mathrm{C} 7-\mathrm{H} 7 \mathrm{~A}$ & 109.6 \\
\hline $\mathrm{O} 1-\mathrm{Ag} 1-\mathrm{O} 2$ & $51.95(11)$ & $\mathrm{C} 8-\mathrm{C} 7-\mathrm{H} 7 \mathrm{~B}$ & 109.6 \\
\hline $\mathrm{N} 1{ }^{\mathrm{ii}}-\mathrm{Ag} 1-\mathrm{O} 2$ & $121.93(12)$ & $\mathrm{C} 6-\mathrm{C} 7-\mathrm{H} 7 \mathrm{~B}$ & 109.6 \\
\hline $\mathrm{N} 2^{\mathrm{i}}-\mathrm{Ag} 1-\mathrm{O} 2^{\mathrm{iii}}$ & $101.35(11)$ & $\mathrm{H} 7 \mathrm{~A}-\mathrm{C} 7-\mathrm{H} 7 \mathrm{~B}$ & 108.2 \\
\hline $\mathrm{O} 1-\mathrm{Ag} 1-\mathrm{O} 2^{\mathrm{iii}}$ & $128.04(11)$ & $\mathrm{C} 13-\mathrm{C} 8-\mathrm{C} 9$ & $110.1(3)$ \\
\hline $\mathrm{N} 1^{\mathrm{ii}}-\mathrm{Ag} 1-\mathrm{O} 2^{\mathrm{iii}}$ & $89.79(11)$ & $\mathrm{C} 13-\mathrm{C} 8-\mathrm{C} 7$ & $110.0(3)$ \\
\hline $\mathrm{O} 2-\mathrm{Ag} 1-\mathrm{O} 2^{\mathrm{iii}}$ & $77.22(13)$ & $\mathrm{C} 9-\mathrm{C} 8-\mathrm{C} 7$ & $109.5(3)$ \\
\hline $\mathrm{C} 3-\mathrm{O} 1-\mathrm{Ag} 1$ & $96.0(3)$ & $\mathrm{C} 13-\mathrm{C} 8-\mathrm{H} 8$ & 109.1 \\
\hline $\mathrm{C} 3-\mathrm{O} 2-\mathrm{Ag} 1$ & $89.9(3)$ & $\mathrm{C} 9-\mathrm{C} 8-\mathrm{H} 8$ & 109.1 \\
\hline $\mathrm{C} 1-\mathrm{N} 1-\mathrm{N} 2$ & $106.7(3)$ & $\mathrm{C} 7-\mathrm{C} 8-\mathrm{H} 8$ & 109.1 \\
\hline $\mathrm{C} 1-\mathrm{N} 1-\mathrm{Ag} 1^{\mathrm{iv}}$ & $122.0(2)$ & $\mathrm{C} 4-\mathrm{C} 9-\mathrm{C} 8$ & $108.5(3)$ \\
\hline $\mathrm{N} 2-\mathrm{N} 1-\mathrm{Ag} 1^{\mathrm{iv}}$ & $130.9(2)$ & $\mathrm{C} 4-\mathrm{C} 9-\mathrm{H} 9 \mathrm{~A}$ & 110.0 \\
\hline $\mathrm{C} 2-\mathrm{N} 2-\mathrm{N} 1$ & $107.6(3)$ & $\mathrm{C} 8-\mathrm{C} 9-\mathrm{H} 9 \mathrm{~A}$ & 110.0 \\
\hline $\mathrm{C} 2-\mathrm{N} 2-\mathrm{Ag} 1^{\mathrm{v}}$ & $135.8(2)$ & $\mathrm{C} 4-\mathrm{C} 9-\mathrm{H} 9 \mathrm{~B}$ & 110.0 \\
\hline $\mathrm{N} 1-\mathrm{N} 2-\mathrm{Ag} 1^{\mathrm{v}}$ & $115.7(2)$ & $\mathrm{C} 8-\mathrm{C} 9-\mathrm{H} 9 \mathrm{~B}$ & 110.0 \\
\hline $\mathrm{C} 2-\mathrm{N} 3-\mathrm{C} 1$ & $104.3(3)$ & $\mathrm{H} 9 \mathrm{~A}-\mathrm{C} 9-\mathrm{H} 9 \mathrm{~B}$ & 108.4 \\
\hline $\mathrm{C} 2-\mathrm{N} 3-\mathrm{C} 4$ & $128.9(3)$ & $\mathrm{C} 4-\mathrm{C} 10-\mathrm{C} 11$ & $108.6(3)$ \\
\hline $\mathrm{C} 1-\mathrm{N} 3-\mathrm{C} 4$ & $126.8(3)$ & $\mathrm{C} 4-\mathrm{C} 10-\mathrm{H} 10 \mathrm{~A}$ & 110.0 \\
\hline $\mathrm{N} 1-\mathrm{C} 1-\mathrm{N} 3$ & $111.0(3)$ & $\mathrm{C} 11-\mathrm{C} 10-\mathrm{H} 10 \mathrm{~A}$ & 110.0 \\
\hline $\mathrm{N} 1-\mathrm{C} 1-\mathrm{H} 1$ & 124.5 & $\mathrm{C} 4-\mathrm{C} 10-\mathrm{H} 10 \mathrm{~B}$ & 110.0 \\
\hline $\mathrm{N} 3-\mathrm{C} 1-\mathrm{H} 1$ & 124.5 & $\mathrm{C} 11-\mathrm{C} 10-\mathrm{H} 10 \mathrm{~B}$ & 110.0 \\
\hline $\mathrm{N} 2-\mathrm{C} 2-\mathrm{N} 3$ & $110.3(3)$ & $\mathrm{H} 10 \mathrm{~A}-\mathrm{C} 10-\mathrm{H} 10 \mathrm{~B}$ & 108.4 \\
\hline $\mathrm{N} 2-\mathrm{C} 2-\mathrm{H} 2$ & 124.8 & $\mathrm{C} 13-\mathrm{C} 11-\mathrm{C} 12$ & $110.0(3)$ \\
\hline $\mathrm{N} 3-\mathrm{C} 2-\mathrm{H} 2$ & 124.8 & $\mathrm{C} 13-\mathrm{C} 11-\mathrm{C} 10$ & $109.2(3)$ \\
\hline $\mathrm{O} 2-\mathrm{C} 3-\mathrm{O} 1$ & $122.1(4)$ & $\mathrm{C} 12-\mathrm{C} 11-\mathrm{C} 10$ & $109.3(3)$ \\
\hline $\mathrm{O} 2-\mathrm{C} 3-\mathrm{C} 6$ & $119.7(4)$ & $\mathrm{C} 13-\mathrm{C} 11-\mathrm{H} 11$ & 109.4 \\
\hline $\mathrm{O} 1-\mathrm{C} 3-\mathrm{C} 6$ & $118.2(4)$ & $\mathrm{C} 12-\mathrm{C} 11-\mathrm{H} 11$ & 109.4 \\
\hline $\mathrm{N} 3-\mathrm{C} 4-\mathrm{C} 9$ & $109.1(3)$ & $\mathrm{C} 10-\mathrm{C} 11-\mathrm{H} 11$ & 109.4 \\
\hline $\mathrm{N} 3-\mathrm{C} 4-\mathrm{C} 10$ & $109.1(3)$ & $\mathrm{C} 11-\mathrm{C} 12-\mathrm{C} 6$ & $110.4(3)$ \\
\hline $\mathrm{C} 9-\mathrm{C} 4-\mathrm{C} 10$ & $110.5(3)$ & $\mathrm{C} 11-\mathrm{C} 12-\mathrm{H} 12 \mathrm{~A}$ & 109.6 \\
\hline $\mathrm{N} 3-\mathrm{C} 4-\mathrm{C} 5$ & $108.4(3)$ & $\mathrm{C} 6-\mathrm{C} 12-\mathrm{H} 12 \mathrm{~A}$ & 109.6 \\
\hline $\mathrm{C} 9-\mathrm{C} 4-\mathrm{C} 5$ & $110.2(3)$ & $\mathrm{C} 11-\mathrm{C} 12-\mathrm{H} 12 \mathrm{~B}$ & 109.6 \\
\hline $\mathrm{C} 10-\mathrm{C} 4-\mathrm{C} 5$ & $109.5(3)$ & $\mathrm{C} 6-\mathrm{C} 12-\mathrm{H} 12 \mathrm{~B}$ & 109.6 \\
\hline $\mathrm{C} 4-\mathrm{C} 5-\mathrm{C} 6$ & $109.5(3)$ & $\mathrm{H} 12 \mathrm{~A}-\mathrm{C} 12-\mathrm{H} 12 \mathrm{~B}$ & 108.1 \\
\hline
\end{tabular}




\begin{tabular}{|c|c|c|c|}
\hline $\mathrm{C} 4-\mathrm{C} 5-\mathrm{H} 5 \mathrm{~A}$ & 109.8 & $\mathrm{C} 8-\mathrm{C} 13-\mathrm{C} 11$ & $109.2(3)$ \\
\hline $\mathrm{C} 6-\mathrm{C} 5-\mathrm{H} 5 \mathrm{~A}$ & 109.8 & $\mathrm{C} 8-\mathrm{C} 13-\mathrm{H} 13 \mathrm{~A}$ & 109.8 \\
\hline $\mathrm{C} 4-\mathrm{C} 5-\mathrm{H} 5 \mathrm{~B}$ & 109.8 & $\mathrm{C} 11-\mathrm{C} 13-\mathrm{H} 13 \mathrm{~A}$ & 109.8 \\
\hline $\mathrm{C} 6-\mathrm{C} 5-\mathrm{H} 5 \mathrm{~B}$ & 109.8 & $\mathrm{C} 8-\mathrm{C} 13-\mathrm{H} 13 \mathrm{~B}$ & 109.8 \\
\hline $\mathrm{H} 5 \mathrm{~A}-\mathrm{C} 5-\mathrm{H} 5 \mathrm{~B}$ & 108.2 & $\mathrm{C} 11-\mathrm{C} 13-\mathrm{H} 13 \mathrm{~B}$ & 109.8 \\
\hline $\mathrm{C} 7-\mathrm{C} 6-\mathrm{C} 12$ & $108.7(3)$ & $\mathrm{H} 13 \mathrm{~A}-\mathrm{C} 13-\mathrm{H} 13 \mathrm{~B}$ & 108.3 \\
\hline $\mathrm{C} 7-\mathrm{C} 6-\mathrm{C} 3$ & $112.6(3)$ & $\mathrm{O} 4^{\mathrm{vi}}-\mathrm{O} 4-\mathrm{H} 2 \mathrm{~W}$ & 84.2 \\
\hline $\mathrm{C} 12-\mathrm{C} 6-\mathrm{C} 3$ & $110.2(3)$ & $\mathrm{O} 44^{\mathrm{vi}}-\mathrm{O} 4-\mathrm{H} 3 \mathrm{~W}$ & 133.4 \\
\hline $\mathrm{C} 7-\mathrm{C} 6-\mathrm{C} 5$ & $109.1(3)$ & $\mathrm{H} 2 \mathrm{~W}-\mathrm{O} 4-\mathrm{H} 3 \mathrm{~W}$ & 108.4 \\
\hline $\mathrm{C} 12-\mathrm{C} 6-\mathrm{C} 5$ & $108.1(3)$ & & \\
\hline $\mathrm{C} 1-\mathrm{N} 1-\mathrm{N} 2-\mathrm{C} 2$ & $-0.1(4)$ & $\mathrm{O} 2-\mathrm{C} 3-\mathrm{C} 6-\mathrm{C} 5$ & $-114.4(4)$ \\
\hline $\mathrm{Ag} 1^{\mathrm{iv}}-\mathrm{N} 1-\mathrm{N} 2-\mathrm{C} 2$ & $173.7(3)$ & $\mathrm{O} 1-\mathrm{C} 3-\mathrm{C} 6-\mathrm{C} 5$ & $65.8(4)$ \\
\hline $\mathrm{C} 1-\mathrm{N} 1-\mathrm{N} 2-\mathrm{Ag} 1^{\mathrm{v}}$ & $171.1(2)$ & $\mathrm{C} 4-\mathrm{C} 5-\mathrm{C} 6-\mathrm{C} 7$ & $58.0(4)$ \\
\hline $\mathrm{Ag} 1^{\mathrm{iv}}-\mathrm{N} 1-\mathrm{N} 2-\mathrm{Ag} 1^{\mathrm{v}}$ & $-15.1(4)$ & $\mathrm{C} 4-\mathrm{C} 5-\mathrm{C} 6-\mathrm{C} 12$ & $-60.0(4)$ \\
\hline $\mathrm{N} 2-\mathrm{N} 1-\mathrm{C} 1-\mathrm{N} 3$ & $0.4(4)$ & $\mathrm{C} 4-\mathrm{C} 5-\mathrm{C} 6-\mathrm{C} 3$ & $-179.3(3)$ \\
\hline 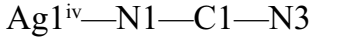 & $-174.1(2)$ & $\mathrm{C} 12-\mathrm{C} 6-\mathrm{C} 7-\mathrm{C} 8$ & $58.8(4)$ \\
\hline $\mathrm{C} 2-\mathrm{N} 3-\mathrm{C} 1-\mathrm{N} 1$ & $-0.5(4)$ & $\mathrm{C} 3-\mathrm{C} 6-\mathrm{C} 7-\mathrm{C} 8$ & $-178.8(3)$ \\
\hline $\mathrm{C} 4-\mathrm{N} 3-\mathrm{C} 1-\mathrm{N} 1$ & $-178.4(3)$ & $\mathrm{C} 5-\mathrm{C} 6-\mathrm{C} 7-\mathrm{C} 8$ & $-58.8(4)$ \\
\hline $\mathrm{N} 1-\mathrm{N} 2-\mathrm{C} 2-\mathrm{N} 3$ & $-0.2(4)$ & $\mathrm{C} 6-\mathrm{C} 7-\mathrm{C} 8-\mathrm{C} 13$ & $-60.3(4)$ \\
\hline $\mathrm{Ag} 11^{\mathrm{v}}-\mathrm{N} 2-\mathrm{C} 2-\mathrm{N} 3$ & $-168.8(3)$ & $\mathrm{C} 6-\mathrm{C} 7-\mathrm{C} 8-\mathrm{C} 9$ & $60.7(4)$ \\
\hline $\mathrm{C} 1-\mathrm{N} 3-\mathrm{C} 2-\mathrm{N} 2$ & $0.4(4)$ & $\mathrm{N} 3-\mathrm{C} 4-\mathrm{C} 9-\mathrm{C} 8$ & $180.0(3)$ \\
\hline $\mathrm{C} 4-\mathrm{N} 3-\mathrm{C} 2-\mathrm{N} 2$ & $178.3(3)$ & $\mathrm{C} 10-\mathrm{C} 4-\mathrm{C} 9-\mathrm{C} 8$ & $-60.1(4)$ \\
\hline $\mathrm{Ag} 1-\mathrm{O} 2-\mathrm{C} 3-\mathrm{O} 1$ & $-4.0(4)$ & $\mathrm{C} 5-\mathrm{C} 4-\mathrm{C} 9-\mathrm{C} 8$ & $61.1(4)$ \\
\hline $\mathrm{Ag} 1-\mathrm{O} 2-\mathrm{C} 3-\mathrm{C} 6$ & $176.3(3)$ & $\mathrm{C} 13-\mathrm{C} 8-\mathrm{C} 9-\mathrm{C} 4$ & $59.9(4)$ \\
\hline $\mathrm{Ag} 1-\mathrm{O} 1-\mathrm{C} 3-\mathrm{O} 2$ & $4.2(4)$ & $\mathrm{C} 7-\mathrm{C} 8-\mathrm{C} 9-\mathrm{C} 4$ & $-61.1(4)$ \\
\hline $\mathrm{Ag} 1-\mathrm{O} 1-\mathrm{C} 3-\mathrm{C} 6$ & $-176.0(3)$ & $\mathrm{N} 3-\mathrm{C} 4-\mathrm{C} 10-\mathrm{C} 11$ & $-179.7(3)$ \\
\hline $\mathrm{C} 2-\mathrm{N} 3-\mathrm{C} 4-\mathrm{C} 9$ & $170.9(3)$ & $\mathrm{C} 9-\mathrm{C} 4-\mathrm{C} 10-\mathrm{C} 11$ & $60.4(4)$ \\
\hline $\mathrm{C} 1-\mathrm{N} 3-\mathrm{C} 4-\mathrm{C} 9$ & $-11.7(5)$ & $\mathrm{C} 5-\mathrm{C} 4-\mathrm{C} 10-\mathrm{C} 11$ & $-61.2(4)$ \\
\hline $\mathrm{C} 2-\mathrm{N} 3-\mathrm{C} 4-\mathrm{C} 10$ & $50.1(5)$ & $\mathrm{C} 4-\mathrm{C} 10-\mathrm{C} 11-\mathrm{C} 13$ & $-60.1(4)$ \\
\hline $\mathrm{C} 1-\mathrm{N} 3-\mathrm{C} 4-\mathrm{C} 10$ & $-132.5(3)$ & $\mathrm{C} 4-\mathrm{C} 10-\mathrm{C} 11-\mathrm{C} 12$ & $60.4(4)$ \\
\hline $\mathrm{C} 2-\mathrm{N} 3-\mathrm{C} 4-\mathrm{C} 5$ & $-69.0(4)$ & $\mathrm{C} 13-\mathrm{C} 11-\mathrm{C} 12-\mathrm{C} 6$ & $59.3(4)$ \\
\hline $\mathrm{C} 1-\mathrm{N} 3-\mathrm{C} 4-\mathrm{C} 5$ & $108.3(4)$ & $\mathrm{C} 10-\mathrm{C} 11-\mathrm{C} 12-\mathrm{C} 6$ & $-60.6(4)$ \\
\hline $\mathrm{N} 3-\mathrm{C} 4-\mathrm{C} 5-\mathrm{C} 6$ & $-179.3(3)$ & $\mathrm{C} 7-\mathrm{C} 6-\mathrm{C} 12-\mathrm{C} 11$ & $-58.4(4)$ \\
\hline $\mathrm{C} 9-\mathrm{C} 4-\mathrm{C} 5-\mathrm{C} 6$ & $-60.0(3)$ & $\mathrm{C} 3-\mathrm{C} 6-\mathrm{C} 12-\mathrm{C} 11$ & $177.8(3)$ \\
\hline $\mathrm{C} 10-\mathrm{C} 4-\mathrm{C} 5-\mathrm{C} 6$ & $61.8(4)$ & $\mathrm{C} 5-\mathrm{C} 6-\mathrm{C} 12-\mathrm{C} 11$ & $59.8(4)$ \\
\hline $\mathrm{O} 2-\mathrm{C} 3-\mathrm{C} 6-\mathrm{C} 7$ & $6.2(5)$ & $\mathrm{C} 9-\mathrm{C} 8-\mathrm{C} 13-\mathrm{C} 11$ & $-60.8(4)$ \\
\hline $\mathrm{O} 1-\mathrm{C} 3-\mathrm{C} 6-\mathrm{C} 7$ & $-173.6(3)$ & $\mathrm{C} 7-\mathrm{C} 8-\mathrm{C} 13-\mathrm{C} 11$ & $59.9(4)$ \\
\hline $\mathrm{O} 2-\mathrm{C} 3-\mathrm{C} 6-\mathrm{C} 12$ & $127.7(4)$ & $\mathrm{C} 12-\mathrm{C} 11-\mathrm{C} 13-\mathrm{C} 8$ & $-59.4(4)$ \\
\hline $\mathrm{O} 1-\mathrm{C} 3-\mathrm{C} 6-\mathrm{C} 12$ & $-52.1(4)$ & $\mathrm{C} 10-\mathrm{C} 11-\mathrm{C} 13-\mathrm{C} 8$ & $60.5(4)$ \\
\hline
\end{tabular}

Symmetry codes: (i) $-x+1 / 2, y-1 / 2,-z+1 / 2$; (ii) $x+1 / 2, y-1 / 2, z$; (iii) $x,-y,-z+1$; (iv) $x-1 / 2, y+1 / 2, z$; (v) $-x+1 / 2, y+1 / 2,-z+1 / 2$; (vi) $-x+1, y,-z+1 / 2$.

Hydrogen-bond geometry $\left(\AA,{ }^{\circ}\right)$

\begin{tabular}{lllll}
\hline$D-\mathrm{H} \cdots A$ & $D-\mathrm{H}$ & $\mathrm{H} \cdots A$ & $D \cdots A$ & $D-\mathrm{H} \cdots A$ \\
\hline $\mathrm{O} 3-\mathrm{H} 1 W \cdots \mathrm{O} 1$ & 0.85 & 1.97 & $2.818(5)$ & 171 \\
$\mathrm{O} 4-\mathrm{H} 2 W \cdots \mathrm{O} 3$ & 0.85 & 1.92 & $2.746(10)$ & 163
\end{tabular}




$\begin{array}{lllll}\mathrm{O} 4-\mathrm{H} 3 W \cdots \mathrm{O} 5 A^{\text {vii }} & 0.85 & 1.81 & 2.56(2) & 147 \\ \mathrm{O} 4-\mathrm{H} 3 W \cdots \mathrm{O} 5 B^{\text {vii }} & 0.85 & 2.11 & 2.83(3) & 143 \\ \mathrm{C} 1-\mathrm{H} 1 \cdots \mathrm{O} 1^{\text {viii }} & 0.94 & 2.43 & 3.336(5) & 162 \\ \mathrm{C} 2-\mathrm{H} 2 \cdots \mathrm{O} 44^{\text {vi }} & 0.94 & 2.58 & 3.516(12) & 171\end{array}$

Symmetry codes: (vi) $-x+1, y,-z+1 / 2$; (vii) $x+1 / 2,-y+1 / 2,-z+1$; (viii) $x-1 / 2,-y+1 / 2,-z+1$. 